\title{
Topological characterization of delocalization in a spin-orbit coupling system
}

\author{
D. N. Sheng and Z. Y. Weng \\ Texas Center for Superconductivity and Department of Physics, University of Houston, Houston, Texas 77204-5506
}

(Received 21 June 1996)

\begin{abstract}
We show that wave functions in a two-dimensional (2D) electron system with spin-orbit coupling can be characterized by a topological quantity-the Chern integer due to the existence of the intrinsic Kramers degeneracy. The localization-delocalization transition in such a system is studied in terms of such a Chern number description, which reproduces the known metal-insulator transition point. The present work suggests a unified picture for various known 2D delocalization phenomena based on the same topological characterization. [S0163-1829(96)51140-9]
\end{abstract}

The metal-insulator phase transition problem in two dimensions has attracted a lot of attention in recent years. With time-reversal and spin rotational symmetries, all states in a two-dimensional (2D) noninteracting electron system should be localized due to disorders, according to the Anderson localization. ${ }^{1,2}$ In contrast to such so-called orthogonal systems, extended states exist in quantum Hall effect (QHE) systems $^{3,4}$ to carry current going through whole samples. These systems in which the time-reversal symmetry is broken by external magnetic fields are known as the unitary class. There are still on-going debates ${ }^{5-7}$ about whether localization-delocalization transition could happen in a random-magnetic-field case. For a time-reversal invariant spin-orbit coupling system, analytic and numerical studies $^{8-10}$ have been given consistent conclusions about the existence of a metal-insulator transition in this so-called symplectic class.

These 2D quantum systems have been classified as three different universality classes, ${ }^{11}$ namely, orthogonal, unitary, and symplectic, in terms of symmetries of Hamiltonians. It is generally believed that symmetries of these systems may play important roles in determining different localizationdelocalization properties. But it is an interesting open question whether 2D localization-delocalization transitions in different symmetry classes are intrinsically different, or whether all of them (both unitary and symplectic classes) are due to the same universal reason.

In the presence of magnetic fields, Thouless and co-workers ${ }^{12}$ and others ${ }^{13,14}$ have shown that the delocalization property of a wave function is well characterized by its associated Chern number (which is equal to the quantizedHall conductance in the unit of $\left.e^{2} / h\right)$. As a topological invariant integer, the Chern number represents a nontrivial topology of wave function. It has been shown ${ }^{14}$ that nodes of an eigenstate wave function with nonzero Chern number can move freely to cover the whole real space when one continuously changes the boundary condition. Thus a nonzero Chern number describes the extensiveness of a wave function. In contrast, a state with zero Chern number should always be localized in two dimensions due to Anderson localization. ${ }^{12}$ Numerical calculations ${ }^{15}$ based on Chern numbers have excellently verified the localization-delocalization phase diagram in the QHE system. In random-magnetic-field systems, even though the total Hall conductance becomes zero on average, the nonzero Chern integer (or quantized Hall conductance) for single-electron states can still exist, and recently a metal-insulator transition has been revealed ${ }^{7}$ numerically in terms of the classification of Chern integers.

It is natural for one to ask if the delocalization property in spin-orbit coupling systems can be similarly understood in terms of such a topological description. Without uniform or random magnetic field, one may expect a zero Chern number and thus a trivial topology for wave functions in their parameter space. However, this is not necessarily correct. According to Berry, ${ }^{16}$ when one adiabatically changes a wave function in its parameter space, the Berry phase of the wave function can exhibit a monopolelike topological structure if there is a twofold degeneracy. In the spin-orbit coupling system, there is a well-known twofold Kramers degeneracy of eigenstates (even in the presence of random scattering) due to the time-reversal invariance. In this paper, we analytically show that such a degeneracy indeed leads to a nontrivial topological classification of wave functions in a spin-orbit coupling system which is essentially the same as in the unitary class cases, and thus one can relate the delocalization property in this system to its topological characteristics. Numerically we demonstrate that a topological transition happens at the point where the density of nonzero Chern numbers become vanishing when the system is extrapolated to infinity, and such a transition indeed coincides with the known critical point of metal-insulator transition obtained based on conventional numerical methods. ${ }^{10}$ Therefore, a unified picture underlying the localization-delocalization transition in 2D systems (both unitary and symplectic classes) is established where all the delocalizations can be essentially characterized by the same topological quantitythe Chern integer.

We start with a tight-binding lattice model of noninteracting electrons with spin-orbit coupling. The Hamiltonian is defined as follows: ${ }^{10}$

$$
\begin{aligned}
H= & -t \sum_{\tau=\hat{x}, \hat{y}} \sum_{i} \hat{c}_{i}^{+} \hat{c}_{i+\tau}-i V \sum_{i} \hat{c}_{i}^{+} \sigma_{y} \hat{c}_{i+\hat{x}} \\
& +i V \sum_{i} \hat{c}_{i}^{+} \sigma_{x} \hat{c}_{i+\hat{y}}+\frac{1}{2} \sum_{i} w_{i} \hat{c}_{i}^{+} \hat{c}_{i}+\text { H.c. }
\end{aligned}
$$


Here $\hat{c}_{i}^{+}=\left(c_{i \uparrow}^{+}, c_{i \downarrow}^{+}\right)$are fermionic creation operators, with $t$ as the nearest-neighbor hopping integral and $V$ as the hopping-spin-flipping (i.e., spin-orbit coupling) term. The strength of spin-orbit coupling is represented by a dimensionless parameter $S=V / \sqrt{t^{2}+V^{2}}$. And $w_{i}$ is a random potential with strength $\left|w_{i}\right| \leqslant W / 2$ ( $W$ is adjustable). For simplicity, we assume no correlations among different sites for $w_{i}$ (i.e., white noise limit). Such a system will be studied under a generalized boundary condition: $\hat{\Psi}\left(i+L_{j}\right)=$ $e^{i \theta_{j}} \hat{\Psi}(i)$ with square lattice width $L_{1}=L_{2}=L$ and a total number of lattice sites $N=L \times L$ (here $\hat{\Psi}$ includes two spin components and $j=1,2$ represent $x$ and $y$ directions, respectively).

Eigenstates of $H$ may be regarded as functions of boundary phases $\theta_{1}$ and $\theta_{2}$, and the topological property of each eigenstate (labeled by $m$ ) can be characterized in terms of relations with $\theta_{1}$ and $\theta_{2}$ through the Chern integer defined as follows: ${ }^{12-14}$

$$
C^{(m)}=\frac{i}{2 \pi} \iint d \theta_{1} d \theta_{2} \sum_{i} F\left(\theta_{1}, \theta_{2} ; i\right),
$$

in which

$$
\begin{aligned}
F\left(\theta_{1}, \theta_{2}, i\right)= & \left\langle\frac{\partial \hat{\Psi}_{m}^{+}\left(\theta_{1}, \theta_{2} ; i\right)}{\partial \theta_{1}} \mid \frac{\partial \hat{\Psi}_{m}\left(\theta_{1}, \theta_{2} ; i\right)}{\partial \theta_{2}}\right\rangle \\
& -\left\langle\frac{\partial \hat{\Psi}_{m}^{+}\left(\theta_{1}, \theta_{2} ; i\right)}{\partial \theta_{2}} \mid \frac{\partial \hat{\Psi}_{m}\left(\theta_{1}, \theta_{2} ; i\right)}{\partial \theta_{1}}\right\rangle,
\end{aligned}
$$

and the area integral in Eq. (2a) covers a $2 \pi \times 2 \pi$ unit cell in the $\theta$ space. The Chern number $C^{(m)}$ in Eq. (2a) can be shown ${ }^{12,13}$ to be an integer. Naively one would expect a zero Chern number $C^{(m)}$ for a system with time-reversal symmetry, since $C^{(m)}$ is proportional to a Hall conductance as mentioned earlier. In fact, one would see cancellation in Eq. (2) due to the symmetry in wave function $\hat{\Psi}$ :

$$
\left(\begin{array}{c}
\Psi_{\uparrow}\left(\theta_{1}, \theta_{2} ; i\right) \\
\Psi_{\downarrow}\left(\theta_{1}, \theta_{2} ; i\right)
\end{array}\right)=\left(\begin{array}{c}
\Psi_{\downarrow}^{*}\left(-\theta_{1},-\theta_{2} ; i\right) \\
-\Psi_{\uparrow}^{*}\left(-\theta_{1},-\theta_{2} ; i\right)
\end{array}\right),
$$

which reflects the time-reversal invariance of the original Hamiltonian. However, in the spin-orbit coupling case, there is always a twofold degeneracy (Kramers degeneracy) in Hamiltonian (1) at boundary conditions with $\theta_{1}, \theta_{2}=\pi \times$ integer. ${ }^{10}$ Both $\hat{\Psi}_{m}$ and its time-reversal counterpart state are degenerate and orthogonal here. Due to such a degeneracy, derivatives in (2b) would become uncertain at the degeneracy points and the contribution from these points needs more careful examination.

In fact, the Chern integer $C^{(m)}$ is equivalent to a Berry phase (divided by $2 \pi$ ) defined on a closed path along the boundary of a unit cell, ${ }^{16}$ which may be converted to a surface integration as in Eq. (2a) by Stokes theorem. Since each degeneracy point in the parameter space will lead to a monopolelike singular contribution to the Berry phase, ${ }^{16}$ a $2 \mathrm{D} \theta$ plane would cut right through such a singularity point. To avoid this difficulty, one has to introduce a third parameter $\epsilon$ to deform the surface integral around the degeneracy points. For example, we may add an infinitesimal term $H_{p}=-\epsilon \Sigma_{i} \hat{c}_{i}^{+} \sigma_{z} \hat{c}_{i}$ to the Hamiltonian. Then the surface integration in Eq. (2a) is understood as the one in a three-dimensional parameter space $\left(\theta_{1}, \theta_{2}, \epsilon\right)$ (here $\epsilon \ll$ $\max \left(t, V, w_{i}\right) / N$ so that it has no effect when one is sufficiently far away from the degeneracy points). Due to the cancellation in the regions far away from the degeneracy points, Eq. (2a) is then reduced to

$$
C^{(m)}=\frac{i}{2 \pi} \sum_{k=1}^{4} \iint_{S_{k}} d \theta_{1} d \theta_{2} \sum_{i} F\left(\theta_{1}, \theta_{2} ; i\right),
$$

in which $S_{k}(k=1-4)$ represent four small surface integrals covering the degeneracy points $(0,0),(\pi, 0),(0, \pi)$, and $(\pi, \pi)$ within a $2 \pi \times 2 \pi$ unit cell. The minimum size of these areas is decided by the strength $\epsilon$ and the final result should not depend on $\epsilon$ at $\epsilon \rightarrow 0^{+}$. One may find that this regularization procedure resembles the one in a magnetic phase transition where an infinitesimal external symmetrybreaking field is usually introduced to lift the ground-state degeneracy for a spontaneous magnetization. Here $H_{p}$ plays a role to lift the Kramers degeneracy such that the hidden topological characterization becomes explicitly shown (see below).

Denote each degeneracy point as $\left(\theta_{1 k}^{0}, \theta_{2 k}^{0}\right)(k=1-4)$ and suppose that eigenstates are already known at these points for a given $\epsilon$. Then one can determine eigenstates at an arbitrary boundary phase $\left(\theta_{1}, \theta_{2}\right)$, which is close enough to $\left(\theta_{1 k}^{0}, \theta_{2 k}^{0}\right)$, by a perturbative method. The following procedure is similar to that in Ref. 17 in treating the levelcrossing problem. The change of the boundary condition may be incorporated into the Hamiltonian as a perturbation, and in terms of the complete set of basis obtained at $\left(\theta_{1 j}^{0}, \theta_{2 j}^{0}\right)$, such a change in Hamiltonian can be generally written as

$$
\Delta H\left(\theta_{1}, \theta_{2}\right)=\left(\begin{array}{cc}
0 & \Delta_{n}^{*} \\
\Delta_{n} & 0
\end{array}\right)
$$

where $2 \times 2$ matrix elements are related to the $(2 n-1)$ th and $(2 n)$ th eigenstates (assuming an energy-increase order for all the eigenstates), which correspond to a pair of twofold degenerate eigenstates of $H$. The off-diagonal terms may be expanded to a leading order of $\left(\theta_{1}-\theta_{1 k}^{0}\right)$ and $\left(\theta_{2}-\theta_{2 k}^{0}\right)$ (if the leading term happens to be zero, higher-order expansions in $\Delta H\left(\theta_{1}, \theta_{2}\right)$ will be needed, and integer Chern numbers can be similarly obtained $\left.{ }^{17}\right)$. The general form is $\Delta_{n}\left(\theta_{1}, \theta_{2}\right)=\alpha_{k}\left(\theta_{1}-\theta_{1 k}^{0}\right)+\beta_{k}\left(\theta_{2}-\theta_{2 k}^{0}\right) \quad$ with $\alpha_{k}=\left(\partial / \partial \theta_{1}\right)\left\langle\Psi_{2 n-1}\left(\theta_{1 k}^{0}, \theta_{2 k}^{0}\right)\left|H+H_{p}\right| \Psi_{2 n}\left(\theta_{1 k}^{0}, \theta_{2 k}^{0}\right)\right\rangle \quad$ and $\beta_{k}=\left(\partial / \partial \theta_{2}\right)\left\langle\Psi_{2 n-1}\left(\theta_{1 k}^{0}, \theta_{2 k}^{0}\right)\left|H+H_{p}\right| \Psi_{2 n}\left(\theta_{1 k}^{0}, \theta_{2 k}^{0}\right)\right\rangle$. Вy diagonalizing the $2 \times 2$ matrix of the Hamiltonian for each $n$, we get the $(2 n-1)$ th and $(2 n)$ th eigenstates at the boundary phase $\left(\theta_{1}, \theta_{2}\right)$ sufficiently close to $\left(\theta_{1 k}^{0}, \theta_{2 k}^{0}\right)$. Then, according to Eq. (3), the Chern numbers for the $(2 n-1)$ th and $(2 n)$ th eigenstates are given by the following integers: ${ }^{17}$

$$
\begin{gathered}
C^{(2 n-1)}=\frac{1}{2} \sum_{j=1}^{4} \operatorname{sgn}\left[\operatorname{Im}\left(\alpha_{j}^{*} \beta_{j}\right)\right] ; \\
C^{(2 n)}=-C^{(2 n-1)},
\end{gathered}
$$




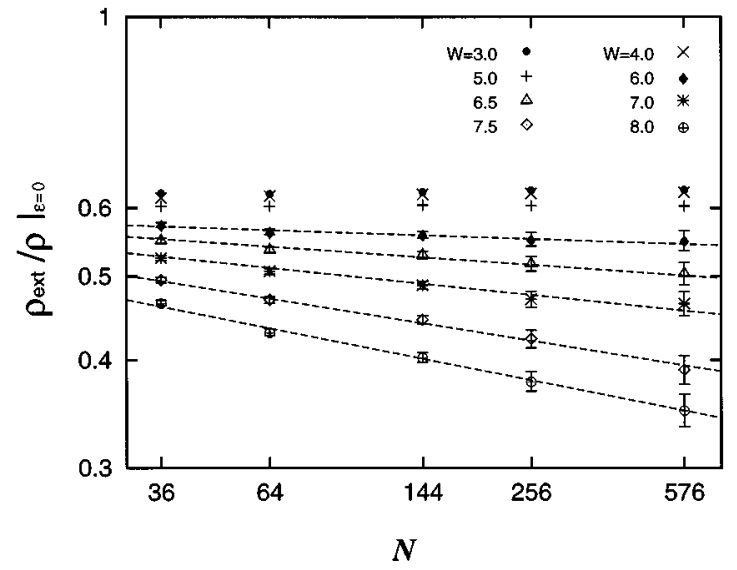

FIG. 1. The ratio of the density of extended states over the total density of states is plotted as a function of sample size $N$ at energy $\varepsilon=0 . W$ is the strength of random scattering. Error bars of the data for $W \leqslant 5.0$ are in the same order of magnitude as those for $W>5.0$ but are not marked in the figure, for clarity.

whose values will not depend on $\epsilon=0^{+}$. \{Here if $\operatorname{Im}\left(\alpha_{j}^{*} \beta_{j}\right)=0$, we define $\operatorname{sgn}\left[\operatorname{Im}\left(\alpha_{j}^{*} \beta_{j}\right)\right]=0$ in (4a).\} Therefore, eigenstates in the spin-orbit coupling system can be indeed classified in terms of nontrivial Chern integers, and two degenerate eigenstates always have Chern numbers with opposite signs such that the total sum of them (and the sum of the Hall conductances) is still zero in consistence with the time-reversal symmetry. Here each degeneracy point gives rise to a contribution similar to a magnetic monopole in unit strength $1 / 2$ as originally discussed by Berry. ${ }^{16}$ Nonzero Chern integers have been used to characterize extended states in both the QHE and random-magnetic-field systems. ${ }^{14,15,7,18}$ By the same token, the delocalization problem in the spin-orbit coupling system should be similarly described here, after the Chern-number classification of wave functions is established. In the following, we shall use this topological characteristics to determine the localizationdelocalization transition in the present system.

We define a density of states $\rho_{\text {ext }}(\varepsilon, N)$ for the eigenstates with nonzero Chern number at energy $\varepsilon$. $\rho_{\text {ext }}$ is related to the density of states for extended states. ${ }^{15,7}$ Exact diagonalization of $H$ is carried out at four boundary conditions where the twofold degeneracy happens, and then Chern numbers are determined through formula (4). The total density of states $\rho(\varepsilon, N)$ and the extended one $\rho_{\text {ext }}(\varepsilon, N)$ are calculated as a function of lattice size $N(N=36,64,144,256$ and 576), which is averaged over random potential configurations (100-5000 random configurations depending on sample sizes) and also averaged over a small energy width $\Delta \varepsilon=0.4 \sqrt{V^{2}+t^{2}}$ in the neighborhood of energy $\varepsilon$ to reduce statistical error. The ratio $\rho_{\text {ext }}(\varepsilon, N) / \rho(\varepsilon, N)$ at the band center $\varepsilon=0$ is presented in Fig. 1 with a $\log -\log$ plot. Different curves correspond to different random strength $W$ 's. The spin-orbit coupling strength $S$ has been chosen to be $S=0.5$ with $\sqrt{V^{2}+t^{2}}=1$ (in order to compare with the results in Ref. 10). The ratio $\rho_{\text {ext }}(\varepsilon=0, N) / \rho(\varepsilon=0, N)$ monotonically decreases as parameter $\mathrm{W}$ increases, which reflects the fact that eigenstates become more and more localized at stronger disorders. For smaller $W(W=3,4$, and 5 as shown

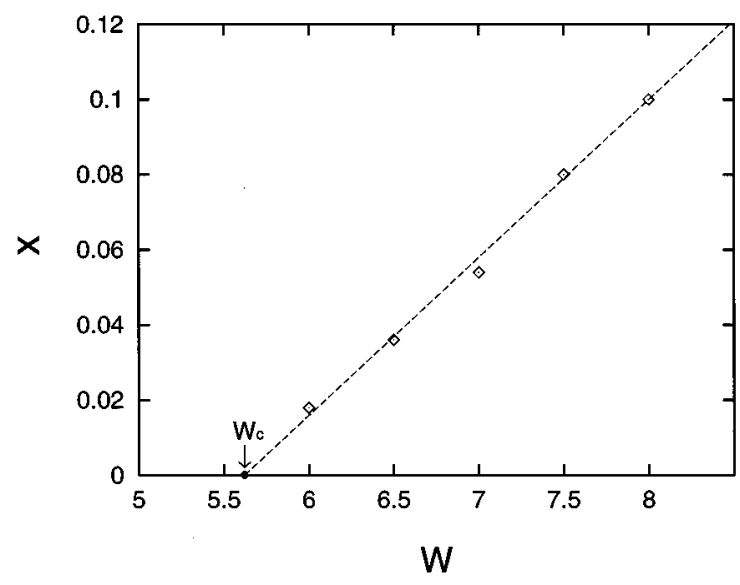

FIG. 2. The exponent $x$ vs the strength of random scattering $W$. The data is extrapolated to $x=0$, giving a critical strength $W_{c}=5.62 \pm 0.08$.

in Fig. 1), the ratio $\rho_{\text {ext }}(\varepsilon=0, N) / \rho(\varepsilon=0, N)$ shows a flat curve with a slight increase at larger sample sizes, suggesting that a finite value of $\rho_{\text {ext }}(\varepsilon=0, N) / \rho(\varepsilon=0, N)$ may be reached if it is extrapolated to infinite lattice size. The trend reverses at larger strengths of random potential, where the ratio $\rho_{\text {ext }}(\varepsilon=0, N) / \rho(\varepsilon=0, N)$ becomes monotonically decreasing with larger sample sizes. In fact, all the data at $W>5$ shown in Fig. 1 can be nicely fit into a straight line, which means $\rho_{\text {ext }}(\varepsilon=0, N) / \rho(\varepsilon=0, N) \propto N^{-x}$ (with $x>0$ to be given below). These finite-size scalings show that at a sufficiently large $W$, the density of states with nonzero Chern number is always extrapolated to zero at large lattices. Similar to the cases in the QHE and random-magnetic-field systems, it means that all the eigenstates here have trivial topology and should be localized.

The exponent $x$ as a function of the disorder strength $W$ is shown in Fig. 2. It is interesting to see that all data fall on a straight line. Thus one can determine the critical strength of disorder $W_{c}$ at $x_{c}=0$ by extrapolation in Fig. 2. Here $W_{c}$ decides a topological phase transition point. At $W>W_{c}$, $\rho_{\text {ext }} \sim N^{-x} \rightarrow 0$ at $N \rightarrow \infty$, while at $W<W_{c}, \rho_{\text {ext }} \rightarrow$ a finite constant at large lattice size. As has been known in the QHE and random-magnetic-field systems, such a topological transition physically corresponds to a localization-delocalization transition. In fact, $W_{c}$ determined from Fig. 2 is approximately $5.62 \pm 0.08$, which is quite close to the value $W_{c}=5.74$ obtained by conventional numerical methods ${ }^{10}$ with much larger lattice sizes $\sim 64 \times 10^{5}$ (a slightly smaller $W_{c}$ in our result may be due to the fact that a finite energy average around $\varepsilon=0$ is made in calculation which could effectively lower $W_{c}$ ).

The above analytic analysis and numerical calculation have shown that delocalization occurs in the spin-orbit coupling case is basically the same as that in the unitary cases. It suggests that all localization-delocalization transitions in 2D noninteracting systems may be universally described as a topological phase transition in terms of the Chern integer. The conventional understanding of metal-insulator transition based on symmetry classes of Hamiltonians may not be essential. For instance, by applying a small but finite magnetic field one may change a spin-orbit coupling system from sym- 
plectic class to unitary class by destroying the time-reversal symmetry. We have checked this case numerically and found ${ }^{19}$ no sudden change in the distribution of Chern integers (thus the topological transition), which actually behaves quite smoothly with small magnetic fields. This fact has already been noticed before in the behavior of localization length. ${ }^{20}$ The universality has also been shown in the study of the critical behavior of metal-insulator transition, where it has been found ${ }^{21}$ that correlation dimensions of spectral measure $\widetilde{D_{2}}$ and fractal eigenstate $D_{2}$ as well as exponent $\eta$ describing the energy correlations of the critical eigenstates in the spin-orbit coupling system are very close to those obtained for the QHE systems. ${ }^{22}$ Experimentally, both in random magnetic field ${ }^{23}$ and spin-orbit coupling ${ }^{24,25}$ systems, magnetoconductances are negative in the delocalized region (here "delocalization" means the localization length is at least larger than the sample size), while positive in the localized region. This common feature can be understood as that in the delocalized region, a finite fraction of eigenstates carry nonzero Chern number and magnetic fields suppress original phase coherence and reduce the number of states having nonzero Chern numbers. ${ }^{19}$ Thus the magnetic field plays a role in reducing delocalization in the "delocalized" region and leads to negative magnetoconductance. In the opposite case, where original wave functions are topologically trivial, mag- netic field will be in favor of delocalization to give rise to a positive magnetoconductance. ${ }^{26}$ This explains why metalinsulator transition is generally accompanied by a sign change of magnetoconductance experimentally in spite of different symmetry classes.

In conclusion, the localization-delocalization transition in a spin-orbit coupling system is studied from a topological point of view. It is shown that wave functions in this timereversal invariant system can be characterized by Chern integers as a result of the intrinsic Kramers degeneracy. We have determined the critical strength of disorder $W_{c}$ for the metal-insulator transition point at energy $\varepsilon=0$ based on such a Chern-integer characterization, which is in excellent agreement with earlier numerical works ${ }^{10}$ by localization-length scalings. Therefore, various known localizationdelocalization transitions in 2D noninteracting systems now can all be described in terms of a topological characterization. Whether there is a far-reaching physical implication behind it needs to be further studied.

The authors would like to thank T. K. Lee and C. S. Ting for helpful discussions. The present work is supported by the Texas Center for Superconductivity at the University of Houston and the Texas Advanced Research Program under Grant No. 3652182.
${ }^{1}$ E. Abrahams, P. W. Anderson, D. C. Licciardello, and V. Ramakrishnan, Phys. Rev. Lett. 42, 673 (1979).

${ }^{2}$ For a review, see P. A. Lee and T. V. Ramakrishnan, Rev. Mod. Phys. 57, 287 (1985).

${ }^{3}$ R. B. Laughlin, Phys. Rev. B 23, 5632 (1981); B. I. Halperin, ibid. 25, 2185 (1982).

${ }^{4}$ H. Levine, S. B. Libby, and A. M. M. Pruisken, Phys. Rev. Lett. 51, 1915 (1983); A. M. M. Pruisken, in The Quantum Hall Effect, edited by R. E. Prange and S. M. Girvin (Springer-Verlag, Berlin, 1990).

${ }^{5}$ S. C. Zhang and D. Arovas, Phys. Rev. Lett. 72, 1886 (1994); V. Kalmeyer and S. C. Zhang, Phys. Rev. B 46, 9889 (1992); Y. Avishai, Y. Hatsugai, and M. Kohmoto, ibid. 47, 9561 (1993); V. Kalmeyer, D. Wei, D. P. Arovas, and S. C. Zhang, ibid. 48, 11095 (1993); D. Z. Liu, X. C. Xie, S. Das Sarma, and S. C. Zhang (unpublished).

${ }^{6}$ A. G. Aronov, A. D. Mirlin, and P. Wolfle, Phys. Rev. B 49, 16609 (1994); T. Sugiyama and N. Nagaosa, Phys. Rev. Lett. 70, 1980 (1993); D. Lee and J. Chalker, ibid. 72, 1510 (1994).

${ }^{7}$ D. N. Sheng and Z. Y. Weng, Phys. Rev. Lett. 75, 2388 (1995).

${ }^{8}$ F. Wegner, Nucl. Phys. B 316, 663 (1989).

${ }^{9}$ S. Hikami, A. I. Larkin, and Y. Nagaoka, Prog. Theor. Phys. 63, 707 (1980); S. Hikami, J. Phys. (Paris) Lett. 46, L719 (1985).

${ }^{10}$ T. Ando, Phys. Rev. B 40, 5325 (1989); A. MacKinnon, in Localization, Interaction and Transport Phenomena, edited by B. Kramer, G. Bergmann, and Y. Bruynseraede (Springer, Berlin, 1985), p. 90; U. Fastenrath et al., Physica A 172, 302 (1991); U.
Fastenrath, ibid. 189, 27 (1992); S. N. Evangelou and T. Ziman, J. Phys. C. 20, L235 (1987); S. N. Evangelou, Phys. Rev. Lett. 75, 2550 (1995).

${ }^{11}$ F. J. Dyson, J. Math. Phys. (N.Y.) 3, 140 (1962); 3, 157 (1962); 3, 166 (1962); 3, 1191 (1962); 3, 1199 (1962).

${ }^{12}$ D. J. Thouless, M. Kohmoto, M. P. Nightingale, and M. den Nijs, Phys. Rev. Lett. 49, 405 (1982); D. J. Thouless, J. Phys. C 17, L325 (1984); Q. Niu, D. J. Thouless, and Y. S. Wu, Phys. Rev. B 31, 3372 (1985).

${ }^{13}$ M. Kohmoto, Ann. Phys. (N.Y.) 160, 343 (1985).

${ }^{14}$ D. P. Arovas et al., Phys. Rev. Lett. 60, 619 (1988).

${ }^{15}$ Y. Huo and R. N. Bhatt, Phys. Rev. Lett. 68, 1375 (1992).

${ }^{16}$ M. V. Berry, Proc. R. Soc. London Ser. A 392, 45 (1984); B. Simon, Phys. Rev. Lett. 51, 2167 (1983).

${ }^{17}$ A.H. MacDonald, Phys. Rev. B 29, 3057 (1984); M. Nielsen and P. Hedegard, ibid. 51, 7679 (1995).

${ }^{18}$ K. Chaltikian, L. Pryadko, and S. C. Zhang, Phys. Rev. B 52, 8688 (1996).

${ }^{19}$ D. N. Sheng and Z. Y. Weng (unpublished).

${ }^{20}$ E. Medina and M. Kardar, Phys. Rev. Lett. 66, 3187 (1991).

${ }^{21}$ L. Schweitzer (unpublished).

${ }^{22}$ J. T. Chalker and G. T. Daniell, Phys. Rev. Lett. 61, 593 (1988); B. Huckenstein and L. Schweitzer, ibid. 72, 713 (1994).

${ }^{23}$ F. B. Mancoff et al. Phys. Rev. B 51, 13269 (1995).

${ }^{24}$ Y. Shapir and Z. Ovadyahu, Phys. Rev. B 40, 12441 (1989).

${ }^{25}$ S. Y. Hsu and J. M. Valles, Jr., Phys. Rev. Lett. 74, 2331 (1995).

${ }^{26}$ G. Bergmann, Phys. Rep. 1, 107 (1984). 